\title{
Equilibrium
}

Quarterly Journal of Economics and Economic Policy

VOLUME 8 ISSUE 4, 2013

ISSN 1689-765X, (Online) ISSN 2353-3293

http://www.equilibrium.umk.pl/

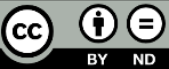

Hanias M., Magafas L., Konstantaki P. (2013), Non Linear Analysis of S\&P Index, "Equilibrium. Quarterly Journal of Economics and Economic Policy", Volume 8, Issue 4, pp. 125-135, DOI: http://dx.doi.org/ 10.12775/EQUIL.2013.030

\author{
Mike Hanias, Lykourgos Magafas \\ Pagania Konstantaki* \\ Kavala Institute of Technology, Greece
}

\section{Non Linear Analysis of S\&P Index}

JEL Classification: $C 53, C 63, G 17$

Keywords: Exchange rates, Time series, Chaos theory.

\begin{abstract}
This paper applies non-linear methods to analyze and predict the daily open $S \& P$ index which is one of the most important stock index in the world. The aim of the analysis is to quantitatively show if the corresponding time series is a deterministic chaotic one and if one or more days ahead prediction can be achieved. These results make the present work a valuable tool for traders investors and funds.
\end{abstract}

\section{Introduction}

Physical models and theories from the field of non-linear Dynamics and are used in order to describe, analyze, explain and solve financial problems. The use of Physical laws in Economics, as well as the use of Physical principles in human behavior will give us additional tools to solve economic problems, model economic systems and predict economic time series as the daily S\&P index. Commonly used and well documented methods, which include nonlinear time series analysis by Kodba et al. (2005), cross correlation by Garas

(C) Copyright Institute of Economic Research \& Polish Economic Society Branch in Torun Date of submission: February 12, 2013; date of acceptance: September 23, 2013

* Contact: lmagafas@otenet.gr, Kavala Institute of Technology, Dept. of Electrical Enginnering, St. Loukas 65404, Kavala, Greece 
and Argyrakis (2007), hierarchal clustering by Coronnello et al. (2007) are presented in the paper.

Alexander and Giblin (1997) enrich the nearest neighbour method by constructing a multivariate nearest neighbour prediction method. Using highfrequency data, they empirically show that the chaos in financial markets can be detected under multivariate model. A broad literature review on multivariate embedding methods can be found in Alexander. Recent evidence on implication of the chaos theory in financial time series prediction comes from the works by Mike P. Hanias. Firstly, Hanias et al. (2006) present a guide of how chaos is used in time series prediction. Hanias et al. (2007) presents evidence on how chaos theory can be used for prediction stock returns in the Athens Stock Exchange. Hanias et al. (2008) also employed chaos theory to show if the Istanbul Stock Exchange has chaotic dynamics. Ozun et al. (2010) uses chaos theory in Athens and Istanbul Stock Exchange, and empirically shows that it has informational efficiency in semi-strong form. In the case when a system presents deterministic chaotic behavior, we can find the number of first order differential equations that described its evolution. The appearance of nonlinear structures is important to the question of out of sample prediction, and this is done in this paper as Hanias and Curtis (2008) shown. For this purpose we have applied the method proposed by Grassberger and Procaccia to evaluate the invariant parameters of S\&P as the correlation and minimum embedding dimensions. Then, we have applied the results from the non linear analysis to predict the corresponding time series with high accuracy.

\section{S\&P Data}

The daily $\mathrm{S} \& \mathrm{P}$ index is presented as a signal $\mathrm{x}=\mathrm{x}(\mathrm{t})$ as it shown at Figure 1 . It covers $\mathrm{N}=5833$ data from $03 / 01 / 1990$ to $25 / 02 / 2013$. The sampling rate was $\Delta \mathrm{t}=1$ day. The data corresponds to closed value every night. 
Figure 2. Time Series of S\&P index

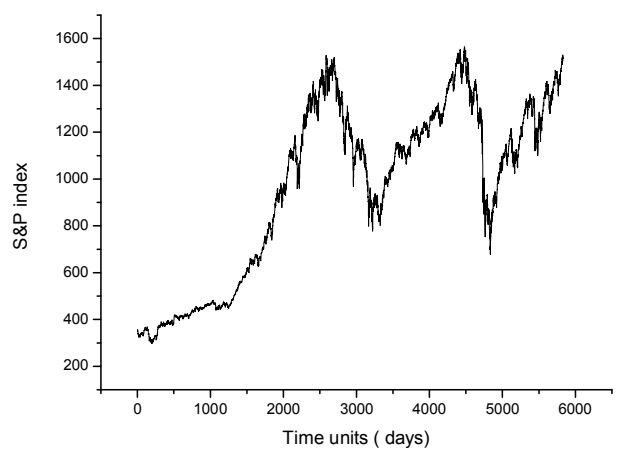

Source: own elaboration.

\section{Theoretical Framework}

In order to evaluate the afore mentioned time series, we have used the method proposed by Grassberger and Procaccia (1983a) and Grassberger and Procaccia (1983b) and successfully applied in similar cases, Hanias and Magafas (2012) and Hanias et.al. (2007). According to Takens (1981) the measured time series was used to reconstruct the original phase space. For this purpose, we calculated the correlation integral, for the recorded signal, defined by the following relation as Kantz and Schreiber (1997) proposed, for $\mathrm{r} \rightarrow 0$ and $\mathrm{N} \rightarrow \infty$,

$$
C(r)=\frac{1}{N_{\text {pairs }}} \sum_{\substack{l=1, j=l+W}}^{N} H\left(r-\left\|\vec{X}_{l}-\vec{X}_{j}\right\|\right)
$$

where:

$\mathrm{N}$ is the number of points,

$\mathrm{H}$ is the Heaviside function,

$$
N_{\text {pairs }}=\frac{2}{(N-m+1)(N-m+W+1)},
$$

$\mathrm{m}$ is the embedding dimension,

The summation counts the number of pairs $\left(\vec{X}_{i}, \vec{X}_{j}\right)$ for which the distance, (Euclidean norm), 
$\left\|\vec{X}_{i}-\vec{X}_{j}\right\|$ is less than $\mathrm{r}$, in an m dimensional Euclidean space.

and $\mathrm{W}$ is the Theiler window. As Theiler pointed out, if temporally correlated points are not neglected, spuriously low dimension estimate may be obtained.

In the above equation, $\mathrm{N}$ is the number of the record data (daily $\mathrm{S} \& \mathrm{P}$ index), here $\mathrm{N}=5833, \vec{X}_{i}$ is a vector in the $\mathrm{m}$ dimensional phase space given by the following relation:

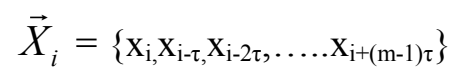

The vector $\vec{X}_{i}=\left\{\mathrm{x}_{\mathrm{i}, \mathrm{X}} \mathrm{X}_{\mathrm{i}-\tau}, \mathrm{x}_{\mathrm{i}-2 \tau}, \ldots . . \mathrm{x}_{\mathrm{i}+(\mathrm{m}-1) \tau}\right\}$, represents a point to the $\mathrm{m}$ dimensional phase space, in which the attractor is embedded each time, where $\tau$ is the time delay determined by the first minimum of the mutual information. In our case $\tau=58$ time steps as shown in Figure 2.

Figure 2. Mutual Information I vs time delay $\tau$

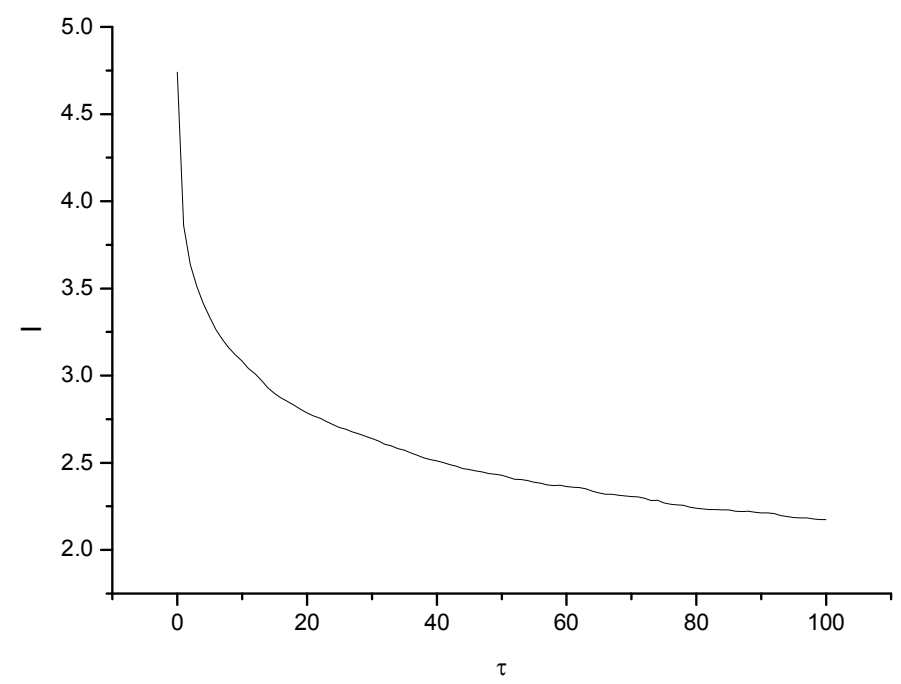

Source: own calculation.

Using the value $\tau=58$ as an optimum delay time for the reconstruction of phase space in eq (1) and also as the Theiler window the same value, we divide this space into hyper cubes with a linear dimension $r$, and we count all 
points with mutual distance less than r. It has been proven by Sprott (2003), Abarbanel (1996) and Ott et.al. (1994) that if our attractor is a strange one, the correlation integral is promotional to $\mathrm{r}^{v}$ where $\mathrm{v}$ is a measure of the dimension of the attractor, called the correlation dimension. Then the correlation function is related to the radius with a power law $C(r) \sim r^{v}$ and $v$ is the slope of the $\log C(\mathrm{r})$ versus logr plot. Since the data set will be continuous, $r$ cannot get to close to zero. To handle this situation, one plots $\log C(\mathrm{r})$ versus $\log$ and selects the apparently linear portion of the graph. The slope of this portion will approximate $v$. Practically, one computes the correlation integral for increasing embedding dimension $\mathrm{m}$ and calculates the related $v(\mathrm{~m})$ in the scaling region.

Using the value $\tau=58$ as an optimum delay time, we reconstruct the phase space. The correlation integral C(r), by definition, is the limit of correlation sum of equation 1 for embedding dimensions $m$ in the range from 1 up to 10 and shown in Figure 3.

Figure 3. Correlation dimension $v$ vs. embedding dimension $m$. The values of $m$ increased from top to bottom

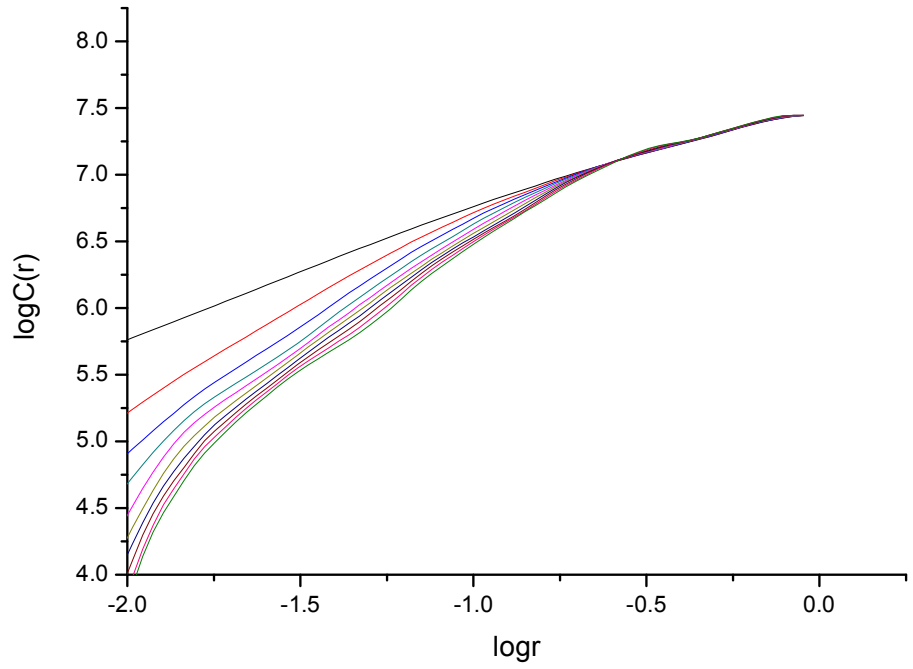

Source: own calculation. 
In Figure 4 the corresponding average slopes $\mathrm{v}$ are given as a function of the embedding dimension $\mathrm{m}$, indicating that for high values of $\mathrm{m}, \mathrm{v}$ tends to saturate at the non integer value of $v=2.3$. The decimal value of $v$ as well as the saturated behavior as a function of $\mathrm{m}$ reveals chaotic behavior of the stock index S\&P.

Figure 4. Correlation dimension $\mathrm{v}$ vs. embedding dimension $\mathrm{m}$

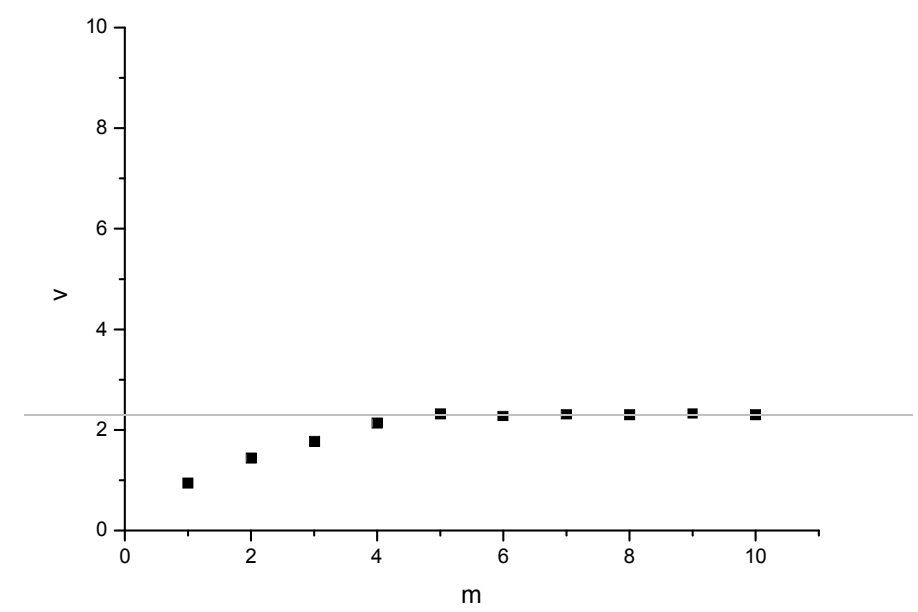

Source: own calculation.

With this value of $\mathrm{v}$, according to Abarbanel (1996), the minimum embedding dimension is $m=3$, so the smallest dimension that contains the attractor is 3 . Sprott (2003), has shown that $2 \mathrm{~m}$ may be sufficient so the topological embedding dimension could have a range from 3 to 6 . So the minimum embedding dimension of the attractor for one to one embedding is 3 4,5 or 6 and it depends on the optimum prediction. The value of $m$ indicates the number of time dependent variables that determine the system evolution.

\section{Quantitative Evidence}

It is well known that chaotic time series is not long term predictable due to their sensitive dependence on initial values. However, it is short-term predictable, and prediction of chaotic time series is very important in real-world applications such as in financial forecasting. Based on the reconstructed state space, one may introduce various approaches for predicting chaotic time series. 
We will apply a simple prediction model that works locally on the reconstructed deterministic data in the state space. It is described fully by Hanias and Curtis (2008). From the signal of equation (2), we can construct an m-dimensional signal $\left\{x_{i}^{m}\right\}=\left\{\left(x_{i}, x_{i+\tau}, x_{i+2 \tau}, \ldots ., x_{i+(m-1) \tau}\right)\right\} \in R^{m}$.

The reconstructed $\mathrm{m}$-dimensional signal projected into the state space can exhibit a range of trajectories, some of which have structures or patterns that can be used for system prediction and modeling. Essentially, in order to predict $\mathrm{k}$ steps into the future from the last $\mathrm{m}$-dimensional vector point $\left\{x_{N}^{m}\right\}$, we have to find all the nearest neighbors $\left\{x_{N N}^{m}\right\}$ in the $\varepsilon$-neighborhood of this point. To be more specific, let $B_{\varepsilon}\left(x_{N}^{m}\right)$ be the set of points within $\varepsilon$ of $\left\{x_{N}^{m}\right\}$ (i.e. the $\varepsilon$-ball). Thus any point in $B_{\varepsilon}\left(x_{N}^{m}\right)$ is closer to the $\left\{x_{N}^{m}\right\}$ than $\varepsilon$. All these points $\left\{x_{N N}^{m}\right\}$ come from the previous trajectories of the system and hence we can follow their evolution k-steps into the future $\left\{x_{N N+k}^{m}\right\}$. The final prediction for the point $\left\{x_{N}^{m}\right\}$ is obtained by local weighted least squares over all neighbors' projections k-steps into the future according to Schouten et.al. (1994), Sugihara and May (1990), Peters (1991) and Ozun et.al. (2007).

We used the values of $\tau$ and $\mathrm{m}$ from our previous analysis so the appropriate time delay $\tau$ was chosen to be $\tau=58$. The embedding dimension $\mathrm{m}=5$ follows from the Taken's embedding theorem, and was used for the state space reconstruction and gives better results than $m=3,4,6$. We choose the optimum number of neighbors to be 9 three times the value of minimum embedding dimension $\mathrm{m}$, as a rule of thumb. In sample actual and predicted time series for $\mathrm{k}=5$, time steps ahead are presented in Figure 5.

Out of sample actual and predicted time series for $\mathrm{k}=5$ time steps ahead are presented in Figure 5. In Table 1, we can compare the predicted values and the actual values for $\mathrm{k}=5$ days ahead. As it is clear from these date of Table 1 and Figure 6, the prediction up to two days is very successful and for the longer time up to five days seems to follow the trend of the index, even though it shows progressive deviation. In any case, this overall possibility makes this study an important tool for investors, traders and professionals of markets. 
Figure 5. In sample actual and predicted time series for $k=1$ time steps ahead

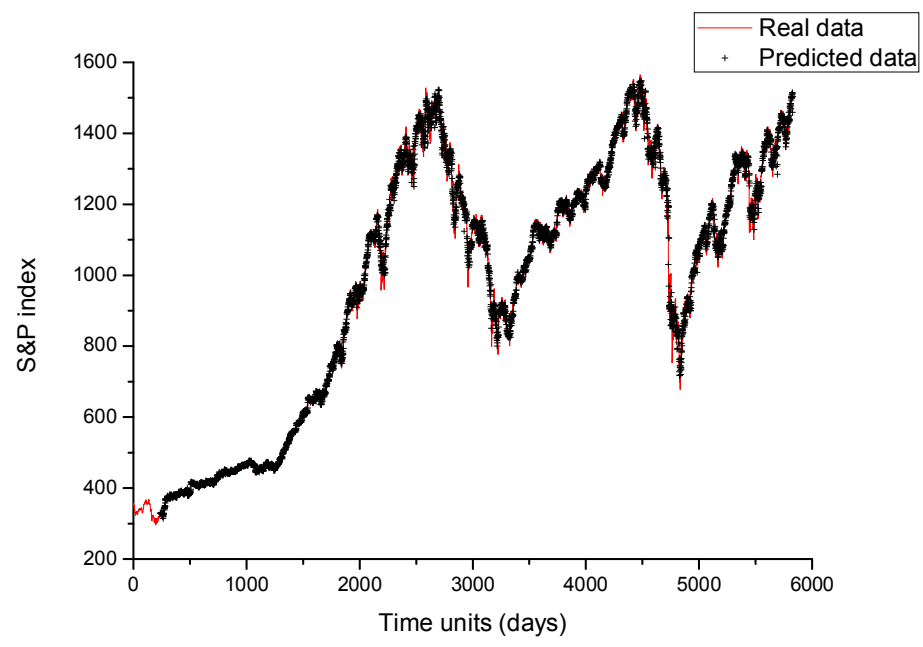

Source: own calculation.

Figure 6. Out of sample Actual and predicted time series for $k=1$ time steps ahead

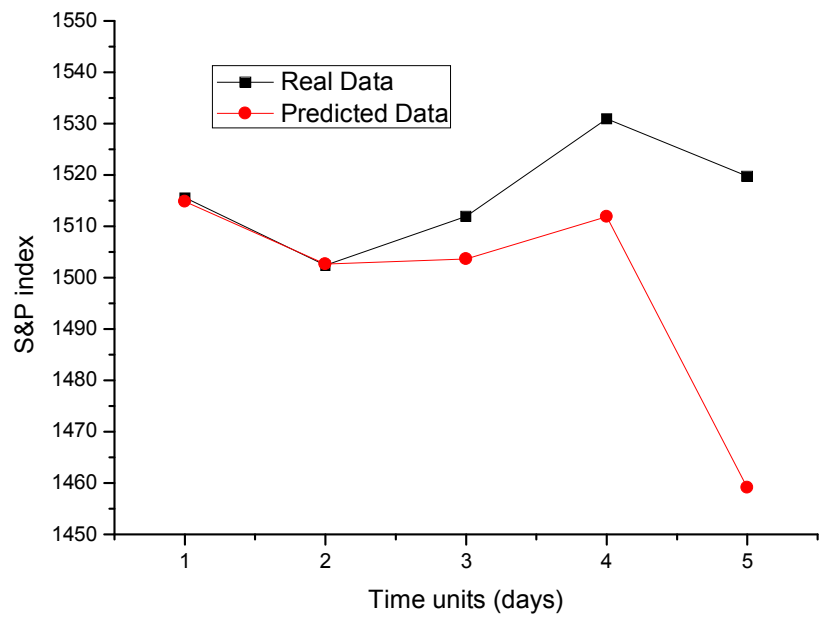

Source: own calculation. 
Table 2. Some original and predicted data (In sample and out of sample)

\begin{tabular}{|r|r|r|}
\hline \multicolumn{1}{|c|}{ Time units } & \multicolumn{1}{c|}{ Original } & \multicolumn{1}{c|}{ Predicted } \\
\hline 5815 & 1498.11 & 1500.88 \\
\hline 5816 & 1501.96 & 1502.58 \\
\hline 5817 & 1507.84 & 1502.02 \\
\hline 5818 & 1517.93 & 1500.41 \\
\hline 5819 & 1509.39 & 1498.47 \\
\hline 5820 & 1512.12 & 1502.56 \\
\hline 5821 & 1511.29 & 1504.04 \\
\hline 5822 & 1495.71 & 1507.49 \\
\hline 5823 & 1487.85 & 1510.34 \\
\hline 5824 & N/A & 1514.8 \\
\hline 5825 & N/A & 1502.64 \\
\hline 5826 & N/A & 1503.66 \\
\hline 5827 & N/A & 1511.86 \\
\hline 5828 & N/A & 1459.1 \\
\hline
\end{tabular}

Source: own calculation.

\section{Conclusions}

In this work, we study the S\&P index using the Chaos Theory. The evaluated results show that the this index follows the Chaos theory, presenting the following characteristics correlation dimension 2.30 and embedding dimension 5. The former proves that the system is a low dimension deterministic and the latter suggests that the number of time dependent variables that determine the system evolution is 5. Using the reconstructed strange attractor, we can predict the future values of S\&P index for two days' time distance.

Finally, the great value of this work consists in the possibility of very accurate predictions of S\&P within two days and to follow the trends up to five days, since in the markets of derivatives (specifically futures, options as well as ETFs) this kind of information is very valuable. We strongly believe that this work is a very useful tool in the markets of derivatives. 


\section{References}

Abarbanel H.D.I (1996), Analysis of observed chaotic data, Springer, New York. Alexander C. (2002), Market models, John Wiley\&Sons Ltd., New York.

Alexander C., Giblin I. (1997), Multivariate embedding methods: Forecasting highfrequency data in the INFFC, "Journal of Computational Intelligence in Finance" Vol. 5 No. 6.

Coronnello C.M., Tumminello F.L., Micciche S. Mantegna R.N. (2007), Economic Sector Identification in a Set of Stocks Traded at the New York Stock Exchange: A Comparative Analysis, "Noise and Stochastics in Complex Systems and Finance", Vol. 6601.

Garas A., Argyrakis P. (2007), Correlation Study of the Athens Stock Exchange, "Physica", Vol. 380.

Grassberger P., Procaccia I. ( 1983a), Estimation of the kolmogorov entropy from a chaotic signal, "Physical Review", Vol. 28, No. 4.

Grassberger P., Procaccia I. (1983b), Measuring the strangeness of strange attractors, "Physica", Vol. 9.

Hanias M.P., Giannaris G., Spyridakis A., Rigas A. (2006), Time series analysis in chaotic diode resonator circuit, "Chaos Solitons and Fractals", Vol. 27, No. 2.

Hanias M.P., Magafas L. (2012), DemoscopoPhysics: A New and Interdisciplinary Research Field, "Chaos and Complexity Theory for Management: Nonlinear Dynamics", Vol. 16.

Hanias M.P., Curtis G., Thallasinos J.E. (2007), Non-linear dynamics and chaos: The case of the price indicator at the Athens Stock Exchange, "International Research Journal of Finance and Economics", Vol. 11.

Hanias M.P., Curtis G., Ozun A. (2008), Chaos theory in predicting the Istanbul Stock Exchange Index, "Empirical Economics Letters", Vol. 7, No. 4.

Hanias M.P., Curtis P.G (2008), Time Series Prediction of Dollar|Euro Exchange Rate Index, "International Research Journal of Finance and Economics", Vol. 15.

Kantz H., Schreiber T. (1997), Nonlinear Time Series Analysis, Cambridge University Press, Cambridge.

Kodba S., Perc M., Marhl M. (2005), Detecting Chaos from a Time Series, "European Journal of Physics", Vol. 26.

Ott E., Sauer T., Yorke J.A. (1994), Coping with chaos, Wiley - Interscience Publication, New York.

Ozun A, Hanias M.P., Curtis P.G. (2010), A chaos analysis for Greek and Turkish equity markets, "EuroMed Journal of Business", Vol. 5, No. 1.

Peters E.E. (1991), Chaos and order in the capital markets, Wiley Finance Editions, New York.

Schouten J.C., Takens F., Bleek C.M. (1994), Estimation of the Dimension of a Noisy Attractor, "Physics Review", Vol. 50, No. 3.

Sprott J.C. (2003), Chaos and Time series Analysis, Oxford University Press. 
Sugihara G., May R.M. (1990), Nonlinear forecasting as a way of distinguishing chaos from measurement error in time series, "Nature", Vol. 344.

Takens F. (1981), Detecting strange attractors in turbulence [in:] Rand D. Young L.-S. (eds.), Dynamical Systems and Turbulence, Lecture Notes in Mathematics, Warwick. 